\title{
A study protocol for a randomized controlled trial of an anti-inflammatory nutritional intervention in patients with fibromyalgia
}

Ana Rita Silva ${ }^{1,2}$ (D) Alexandra Bernardo², Maria Fernanda de Mesquita², José Vaz Patto ${ }^{3}$, Pedro Moreira ${ }^{1,4,5}$, Maria Leonor Silva ${ }^{2}$ and Patrícia Padrão ${ }^{1,4^{*}}$

\begin{abstract}
Background: This study aims to analyze the effects of a potentially anti-inflammatory nutritional intervention in disease assessment parameters, inflammatory markers, and quality of life of fibromyalgia (FM) patients.

Methods: A sample of 100 female patients diagnosed with FM, followed up at Portuguese Institute of Rheumatology (IPR) in Lisbon, is being randomly allocated in two groups. Patients in the intervention group are adopting an anti-inflammatory diet, characterized by the exemption of the intake of foods containing gluten, dairy, sugar, and ultra-processed foods, during 3 months. During the first month, a low fermentable oligo-, di-, and monosaccharides and polyols (FODMAPs) diet is implemented, along with the anti-inflammatory diet, followed by the reintroduction of all fruits and vegetables over a consecutive period of 2 months. Patients in the control group are adopting a diet based on general recommendations for healthy eating. The outcomes are pain, fatigue, quality of sleep, quality of life, gastrointestinal symptoms, and inflammation. Before and after the 3 months intervention, and also 1 month after beginning the intervention, the following questionnaires are applied: Revised Fibromyalgia Impact Questionnaire, visual analog pain scale, Brief Pain Inventory,visual analog scale from a list of common gastrointestinal and extraintestinal symptoms in FM, Short Form 36, Fatigue Severity Survey, and Pittsburg Sleep Quality Index. Ultra-sensitive serum C-reactive protein, eritrocyte sedimentation rate, and interleukin-8 are determined. Age, physical activity, anthropometric parameters, and body composition are being collected. Student's $t$ test will assess the association between the disease evaluation parameters, the inflammatory markers, and the dietary interventions.
\end{abstract}

Discussion: The results of this study are expected to determine whether a change in patient nutrition helps to alleviate symptoms, which would optimize medical intervention.

Trial registration: www.ClinicalTrials.gov NCT04007705. Registered on July 5, 2019.

Keywords: Fibromyalgia, Diet, Anti-inflammatory, FODMAPs, Pain, Quality of life, Randomized controlled trial

\footnotetext{
* Correspondence: patriciapadrao@fcna.up.pt

${ }^{1}$ Faculdade de Ciências da Nutrição e Alimentação, Universidade do Porto, Rua Dr. Roberto Frias, 4200-465 Porto, Portugal

${ }^{4}$ EPIUnit, Instituto de Saúde Pública, Universidade do Porto, Rua das Taipas, $n^{\circ} 135,4050-600$ Porto, Portugal

Full list of author information is available at the end of the article
}

(c) The Author(s). 2021 Open Access This article is licensed under a Creative Commons Attribution 4.0 International License, which permits use, sharing, adaptation, distribution and reproduction in any medium or format, as long as you give appropriate credit to the original author(s) and the source, provide a link to the Creative Commons licence, and indicate if changes were made. The images or other third party material in this article are included in the article's Creative Commons licence, unless indicated otherwise in a credit line to the material. If material is not included in the article's Creative Commons licence and your intended use is not permitted by statutory regulation or exceeds the permitted use, you will need to obtain permission directly from the copyright holder. To view a copy of this licence, visit http://creativecommons.org/licenses/by/4.0/ The Creative Commons Public Domain Dedication waiver (http://creativecommons.org/publicdomain/zero/1.0/) applies to the data made available in this article, unless otherwise stated in a credit line to the data. 


\section{Background}

Fibromyalgia (FM) is a chronic non-degenerative disease of unknown etiology, with a prevalence range between 0.5 and $2 \%$ worldwide [1], 2.1\% (95\% CI 2.0-2.2) in men and 3.6\% (CI 95\% 3.5-3.7) in women [2]. In Portugal, the estimated prevalence is $1.7 \%$ [3]. The main symptoms of the disease are musculoskeletal pain and chronic fatigue, in addition to nonrestorative sleep, morning stiffness, depression, anxiety [1], and gastrointestinal (GI) symptoms similar to irritable bowel syndrome (IBS) [4]. Medical therapy consists mainly in analgesic, muscle relaxants, and non-steroids anti-inflammatory drugs (NSAID), but it seems not to completely resolve the symptoms of the disease $[1,5]$.

Recently, several authors showed an association between FM and dysbiosis [6,7], and in particular with small intestinal bacterial overgrowth (SIBO) $[8,9]$, characterized by the inappropriate colonization of the distal small bowel with colonic bacteria [10]. A clinical trial with 38 FM women showed that a low ingestion in fermentable oligo, di-, and monosaccharides and polyols (FODMAPs) could improve SIBO, decreasing pain associated with FM, fatigue, gastric pain, and intestinal changes after 4 weeks [11].

Furthermore, other studies revealed a presence of intestinal inflammation $[4,12-14]$, through a plasma proinflammatory cytokines increase [15-17], particularly interleukin (IL)- 6 and IL-8 $[16,17]$, suggesting a low grade inflammation in these patients, associated with dysbiosis $[6,7]$. Literature suggests that foods with inflammatory potential, as the ones described in "Dietary Inflammatory Index" $[18,19]$ could have a critical role in FM symptoms. Additionally, it is also known the pro-inflammatory effect of gluten [20], dairy [21], and ultra-processed foods [22], and on the other hand, the anti-inflammatory potential of omega 3 [23] and antioxidants [24].

In fact, in a systematic review conducted by our team, it was reported that pain and functional repercussion in FM along with quality of life [25-27], quality of sleep [26], anxiety [27], depression [27, 28], and inflammatory biomarkers [28] seem to improve with a hypocaloric diet $[27,28]$, a raw vegetarian diet $[25,26]$ or a low FODMAPs diet [11]. However, the existing clinical trials on this subject are scarce and low quality, which does not allow conclusions to be drawn [29]. Additionally, to our knowledge, a nutritional approach involving a combination of several anti-inflammatory dietary factors has never been designed.

Taking those findings together, it seems relevant to test the hypothesis that a dietary intervention which includes potentially anti-inflammatory foods and excludes the potentially pro-inflammatory ones, and that simultaneously allows an optimization of the intestinal microbiota, could reduce intestinal inflammation and dysbiosis, and consequently improve the FM patient's reported outcomes (PRO).

\section{Methods and analysis}

The study protocol was developed considering the SPIR IT checklist (Additional file 1) and guidelines and is registered in www.ClinicalTrials.gov (NCT04007705).

The study aims to analyze the effects of a potentially anti-inflammatory and low FODMAPs diet, compared to healthy eating recommendations, in disease assessment parameters, namely pain, fatigue, sleep quality, and GI alterations, in inflammatory markers and quality of life in FM patients.

\section{Study design}

A randomized controlled clinical trial, blind to patients, has started in April 2019 at the Portuguese Institute of Rheumatology [Instituto Português de Rematologia (IPR)] in Lisbon. All women diagnosed with FM followed-up at the IPR, with a medical appointment scheduled between February 2019 and December 2020, are being invited to participate in the study. The recruitment is being performed as the patients are identified in the appointment.

\section{Study setting}

After eligibility criteria confirmation and informed consent applied (Additional file 2), participants are being allocated to intervention or control group. Allocation of participants is performed using systematic procedures. Participants were sequentially assigned to intervention or control group as they were recruited. Due to the nature of the intervention, the allocation of experimental groups is blind to patients but not to researchers, as they will then apply the appropriate dietary plan. Each participant is given a code, to ensure anonymity and confidentiality of collected data.

\section{Sample size}

In order to define the sample size required for the study and to give a statistical power of $80 \%$, G-Power Software version 3.1.9.4 revealed that, for a desirable effect size of $50 \%$, a minimum sample size of 45 individuals is required. In order to prevent follow-up losses, the target sample size is $n=100$.

\section{Participant characteristics and eligibility criteria}

Inclusion criteria are:

1- Female adults, aged over 18 and under 75 years old;

2- Diagnosis of FM performed by the physician, according to the Rome III criteria of the American College of Rheumatology, revised in 2010;

3- Ability to read and sign the informed consent; and 
4- Stable dose therapy within 4 weeks before the study begins.

\section{Exclusion criteria are:}

1- Patients with pathologies that prevent to follow the dietary intervention;

2- Patients currently undergoing lactation or pregnancy;

3- Prior or current clinical history of abuse of drug or other substances;

4- Change of therapy during the intervention period;

5- Presence of other inflammatory diseases; and

6- Uncontrolled medical conditions (e.g., diabetes mellitus, heart disease, renal failure, neoplastic diseases, liver diseases).

\section{Intervention}

Patients are being contacted to schedule the first phase of the study (M0). During the first meeting with the researchers, a blood sample is collected, and the evaluation questionnaires are fulfilled. Additionally, anthropometric parameters (weight, height, and waist perimeter) and body composition are assessed, using a bio-impedance scale. The diet meal plan is determined by an investigator team nutritionist, according to the allocated group, taking into account basal metabolic rate, physical activity, lifestyle, food habits, and preferences of the patient, in order to ensure its feasibility. After 3 months of intervention, patients from both groups meet the researcher in order to perform a new blood collection, to assess weight and body composition and to complete all the evaluation questionnaires.

\section{Dietary interventions specifications}

The intervention group (G1) is adopting an antiinflammatory diet, which is characterized by the exclusion of potentially inflammatory foods, namely gluten, dairy, sugar, and ultra-processed foods, over a consecutive period of 3 months. During the first month, a low FODMAPs diet is being implemented along with the anti-inflammatory diet, followed by the reintroduction of all fruits and vegetables over a consecutive period of 2 months, for a total of 3 months of intervention. The control group (G2) is adopting a diet based on recommendations for healthy eating in accordance with the World Health Organization (WHO) [30]. Both diets are determined by a nutritionist investigator during nutrition consultations using a leaflet, to help compliance. Examples of recipes are being delivered to help patients to comply with the outlined dietary plan. A table of foods to consume and to avoid is being provided to participants belonging to the intervention group during low FODMAPs diet phase (Additional file 3).

\section{Adherence}

During the intervention period, patients are being monitored every 15 days, by telephone, in order to assess compliance and any change regarding the inclusion and exclusion criteria, as well as to clarify any question about the intervention. Biweekly phone contacts are made in order to monitor the compliance with the recommendations. Participants are asked to fulfill a food diary of the 3 previous days to phone contact, and energy, macro, and micronutrients intake are then calculated for both groups. In addition, time of different meals is also analyzed. The Food Processor Software (version 11.2.274) is being used to analyze food records.

The experimental design of the present study is shown schematically in Figs. 1 and 2.

\section{Intervention group}

\section{Anti-inflammatory diet}

The anti-inflammatory diet is characterized by the exclusion of potential inflammatory foods, such as gluten, dairy, free sugar, and ultra-processed food, rich in sugar, hydrogenated fat, and food additives. Despite the controversy surrounding the ingestion of these foods, some authors defend the existence of an association of these with an increase in serum C-reactive protein (CRP) $[18,31,32]$ and various inflammatory diseases [33], including rheumatic diseases [34].

Gluten Some authors describe an association between the characteristic symptoms of FM and the presence of altered intestinal permeability and dysbiosis $[6,7,10]$. In the presence of dysbiosis occurs the destruction of tight juctions, proteins present in enterocytes and responsible for preventing the entry of pathogens. The consequent intestinal hyperpermeability triggers, in turn, an immunological reaction of inflammatory character [35], described by several authors as low grade inflammation [36]. Intestinal hyperpermeability appears to be caused by several factors, including gliadin present in gluten [37-39]. In this way, it would be possible to suggest that the exclusion of gluten could allow a lower prevalence of dysbiosis, and therefore less intestinal inflammation.

Dairy There are several different casein subtypes in milk. In bovine milk, the predominant subtype is $\alpha$ casein (50-55\%), which does not exist in human milk [40], besides $\beta$-casein (35\%) and $\kappa$-casein (15\%). In addition, there are two types of $\beta$-casein, namely A1 and $\mathrm{A} 2$, being the $\mathrm{A} 1 \beta$-casein the prevalent one in Europe dairy products [41]. A systematic review concluded that A1 was associated to a higher prevalence of GI symptoms and increased intestinal inflammation in humans, compared to A2 [42]. The mechanism seems to be related to the activation of the Th2 signaling pathway in the intestine [43], which promote inflammation. 


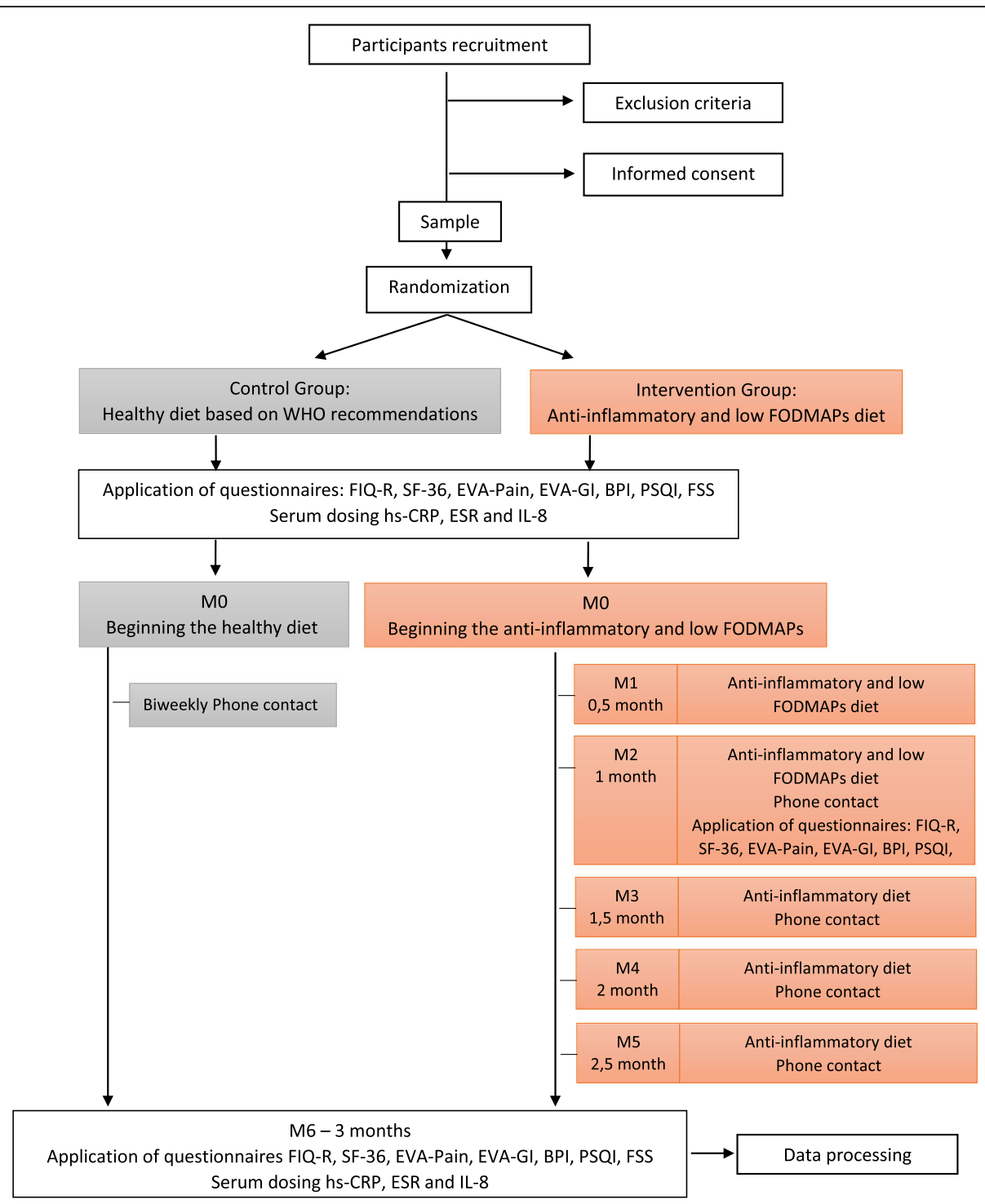

Fig. 1 Experimental design of the randomized controlled clinical trial. Note: FODMAPs, fermentable oligo-, di-, and monosaccharides and polyols; FIQ-R, Revised Fibromyalgia Impact Questionnaire; SF-36, Short Form 36; EVA-Pain, visual analog pain scale; EVA-Gl, visual analog scale from a list of common gastrointestinal and extraintestinal symptoms in FM; BPI, brief pain inventory; PSQI, Pittsburg Sleep Quality Index; FSS, Fatigue Severity Survey; hs-CRP, serum C-reactive protein; ESR, eritrocyte sedimentation rate; IL-8, interleukin-8

Sugar Sugar is a recognizably inflammatory food. In recent years, WHO has been setting up standards for reducing its ingestion. Its excessive consumption promotes the production of free radicals, leading to an increase in oxidative stress [44]. On the other hand, a hyperinsulinogenic environment enhances the expression of proinflammatory molecules [45].

Ultra-processed foods Several authors define ultraprocessed food as potentially inflammatory, mainly due to its free sugars, hydrogenated fat, and food additives content $[46,47]$. Additionally, it is known that its relevant accumulation of advanced glication products (AGEs) is also related to a pro-inflammatory effect [48, 49]. When ingested, AGEs cross the epithelial barrier, attaching to the receptors in the dendritic cells of the mucosa, and promote the uptake of the antigens and to $\mathrm{T}$ cells, specifically Th1, Treg, Th2, and Th17, proinflammatory and inducers of allergic process [50]. AGEs in the cell activate cascades of signaling the production of inflammatory molecules, such as TNF $\alpha$, IL6, and VCAM1 [51]. 


\begin{tabular}{|c|c|c|c|c|c|c|c|c|c|}
\hline \multirow[b]{3}{*}{ TIMEPOINT } & \multirow{3}{*}{$\begin{array}{c}\text { Enrolment } \\
\text { Feb-19 to } \\
\text { Feb-2021 }\end{array}$} & \multicolumn{8}{|c|}{ STUDY PERIOD } \\
\hline & & \multirow{2}{*}{$\begin{array}{c}\text { Allocation } \\
\text { Day } 1\end{array}$} & \multicolumn{6}{|c|}{ Post-allocation } & \multirow{2}{*}{$\begin{array}{r}\text { Close-out } \\
\text { Day } 90\end{array}$} \\
\hline & & & $\begin{array}{c}\text { Day } \\
1\end{array}$ & $\begin{array}{c}\text { Day } \\
15\end{array}$ & $\begin{array}{c}\text { Day } \\
30\end{array}$ & $\begin{array}{c}\text { Day } \\
45\end{array}$ & $\begin{array}{c}\text { Day } \\
60\end{array}$ & $\begin{array}{c}\text { Day } \\
75\end{array}$ & \\
\hline \multicolumn{10}{|l|}{ ENROLMENT: } \\
\hline \multirow[b]{2}{*}{ Informed consent } & $\mathrm{X}$ & & & & & & & & \\
\hline & $\mathrm{X}$ & & & & & & & & \\
\hline Allocation & & $\mathrm{X}$ & & & & & & & \\
\hline \\
\hline \multicolumn{10}{|l|}{$\begin{array}{r}\text { Anti-inflammatory } \\
\text { and Low FODMAPs }\end{array}$} \\
\hline \multirow{2}{*}{\multicolumn{10}{|c|}{$\begin{array}{r}\text { Healthy Diet based } \\
\text { on OMS } \\
\text { recommendations } \\
\text { (control) }\end{array}$}} \\
\hline & & & & & & & & & \\
\hline \multicolumn{10}{|l|}{ ASSESSMENTS: } \\
\hline $\begin{array}{r}\text { Questionnaires: } \\
\text { FIQ-R, SF-36, EVA- } \\
\text { Pain, EVA-GI, BPI, } \\
\text { PSQI, FSS }\end{array}$ & & & $\mathrm{X}$ & & $\mathrm{X}$ & & & & $\mathrm{X}$ \\
\hline Serum dosing hs- & & & $\mathrm{X}$ & & & & & & $\mathrm{X}$ \\
\hline Body Composition & & & $\mathrm{X}$ & & & & & & $\mathrm{X}$ \\
\hline 3-day food records & & & $\mathrm{X}$ & $\mathrm{X}$ & $\mathrm{X}$ & $\mathrm{X}$ & $\mathrm{X}$ & $X$ & $\mathrm{X}$ \\
\hline
\end{tabular}

Fig. 2 Schedule of enrolment, interventions, and assessments, according to SPIRTT guidelines. Note: FODMAPs, fermentable oligo-, di-, and monosaccharides and polyols; FIQ-R, Revised Fibromyalgia Impact Questionnaire; SF-36, Short Form 36; EVA-Pain, visual analog pain scale; EVA-Gl, visual analogue scale from a list of common gastrointestinal and extraintestinal symptoms in FM; BPI, brief pain inventory; PSQI, Pittsburg Sleep Quality Index; FSS, Fatigue Severity Survey; hsCRP, serum C-reactive protein; ESR, eritrocyte sedimentation rate; IL-8, interleukin-8

Anti-inflammatory food components There are some foods with recognized anti-inflammatory potential. Omega 3 , especially at an adequate omega 6:omega 3 ratio, allows the production of prostaglandins, leukotrienes, resolvins, and protectins, which in turn promote the expression of anti-inflammatory cytokines [23]. In that sense, the ingestion of walnuts and omega 3 rich fish, such as tuna, mackerel, sardines, horse mackerel, and salmon, are being encouraged. Additionally, antioxidants in foods are known to decrease the free radicals production, which in turn helps to decrease the oxidative stress and, consequently, the expression of pro-inflammatory molecules [24, 52]. Thus, the intake of foods rich in antioxidants, such as fruit and vegetables, is also being promoted. Thus, the variability in the choice of vegetables and fruits was promoted, in order to obtain several different antioxidants, such as vitamin $\mathrm{C}$ (kiwi, orange), phenolic compounds (black grapes, pomegranate, blackberries, and raspberries), quercitin (apple), zeaxanthin (blueberries), indole-3carbinol (broccoli, cabbage), and vitamin A (pumpkin, carrot, sweet potato). The intake of other foods rich in antioxidants was also promoted, such as cocoa, ginger, and white and green tea $[53,54]$.

Moreover, one of the most important factors in an anti-inflammatory diet is the maintenance of glycemic index, through a greater intake of fibers and suitable proteins and fats, against a balanced intake of carbohydrates. 


\section{Low FODMAPs diet}

The presence of dysbiosis [4, 10, 12], and in particular of SIBO $[8,9]$, has been described in FM patients, with a significant improvement in pain, fatigue, gastric pain, mobility, and GI symptoms, after 4 weeks of low FODMAPs diet [11]. Marsh and colleagues meta-analysis support the efficacy of a diet with a low intake of foods rich in FODMAPs for a period of 4 to 6 weeks in the treatment of GI symptoms, including abdominal pain, abdominal distention, constipation, diarrhea, and flatulence [8], symptoms that are found very often in FM patients [4]. Since this is a recurrent situation in FM [4], it makes sense to start by trying to optimize the quality of the intestinal microbiota, in order to normalize these symptoms, before starting the antiinflammatory diet.

This intervention involves avoiding all dairy; all cereals except rice and oats; cashew; all fruit other than banana, citrus, pineapple, red berries, strawberries, and kiwi; and all vegetables other than pumpkin, cabbage, lettuce, tomato, carrot, and cucumber, for a period of 4 to 6 weeks [8].

\section{Control group}

The control group is receiving a dietary meal plan based on healthy eating recommendations in accordance with WHO guidelines. According to WHO, a healthy diet contains at least $400 \mathrm{~g}$ of fruits and vegetables, excluding potatoes, sweet potatoes, cassava, and starchy roots. A consumption of legumes, nuts, and whole grains (wheat, maize, millet, oats, rice, rye) is also promoted, as well as an intake of less than $5 \mathrm{~g}$ of salt per day, less than $10 \%$ of total energy intake from free sugars and less than $30 \%$ of total energy intake from fats, giving preference to unsaturated fats [55].

\section{Outcome measures}

The primary PRO of interest for this study are pain, fatigue, quality of sleep, quality of life, GI symptoms, and the presence of inflammation. To determine the effect of dietary intervention on the disease, the following questionnaires are being included:

- Revised Fibromyalgia Impact Questionnaire (FIQR) [56], to verify the impact of FM on the patient's life;

- Visual analog pain scale (EVA_Pain) [57], validated by Boonstra and colleagues [58], and brief pain inventory (BPI) to assess pain [59], validated by Keller and colleagues [60];

- Visual analog scale from a list of common gastrointestinal and extraintestinal symptoms in FM, IBS, and non-celiac gluten sensitivity (NCGS) to assess GI symptoms [61], validated by Bengtsson and colleagues [62];

- Short Form 36 (SF-36) [63], to check the quality of life, validated by Fredheim and colleagues [64];

- Validated Fatigue Severity Survey (FSS) [65], to check the fatigue level;

- Validated Pittsburg Sleep Quality Index (PSQI) [66], to check the quality of sleep.

Additionally, serum high-sensitive CRP (hs-CRP), Erythrocyte Sedimentation Rate (ESR) and Interleukin-8 (IL-8) are being measured to assess the presence of inflammation. The serum collection and hs-CRP and ESR analysis is being perfomed by Joaquim Chaves Saúde Laboratory, an external entity. The biomarker IL-8 quantification is being performed according to ImmuliteR® (Siemens, Germany) manufacturer's protocol. Details on collection, laboratory evaluation, and storage of IL-8 is presented on Additional file 4.

Data on age, physical activity, and anthropometric parameters, such as waist circumference, height, and weight, are also being collected. Body composition, specifically fat mass, lean mass, and water, is being assessed by bio-impedance, through the scale of Inbody brand, model 770 .

\section{Patient and public involvement}

Patients or members of the public were not involved in the design, conduct, reporting, or dissemination of the research.

\section{Statistical analysis}

Baseline demographic and clinical characteristics of the participants of both groups will be analyzed using descriptive statistics. For the continuous normal distributed variables, the t-student test will be used to assess the association between the disease evaluation parameters, the inflammatory markers, and the dietary intervention. Correlations between variables will be sought at the different assessment moments. Regression coefficients will be calculated to determine the contribution of the domains for each variable. ANOVA will be used to evaluate the participants' evolution within each group.

Missing data will not be included in the statistical analysis. Participants who discontinue or deviate from intervention protocols, as well as patients who meet exclusion criteria at some point of the intervention period, will be excluded. Motive of exclusion will be the outcome to be collected from these participants.

Statistical analysis will be performed using IBM SPSS Statistics Software, version 19.0. A $p$ value of 0.05 is considered statistically significant. 


\section{Discussion}

The results of this study are expected to determine whether a change in patient nutrition helps to alleviate symptoms, which would optimize medical intervention.

To our knowledge, a nutritional approach involving a combination of several anti-inflammatory dietary factors has never been designed. Nutritional approaches in FM, to date, had always isolated dietary components that are believed to have a negative effect on disease symptoms, such as the application of a gluten-free [67] and aspartame-free diet [68]. An integrative approach has never been undertaken to include anti-inflammatory components and exclude the pro-inflammatory ones.

A recent systematic review (2018) allowed us to determine that dietary interventions seem to be promising as complementary therapies in FM, particularly a hypocaloric diet $[27,28]$, a raw vegetarian diet $[25,26]$, or a low FODMAP diet [11]. However, the studies that exist are of poor quality, according to the Cochrane Risk of Bias [29]. In our study, we intend to increase the sample size and ensure a good completion of nutritional interventions, in order to increase the quality of the study.

The WHO recommendations in the control group, which already could have some positive impact on patients' health, could be a limiting factor in the interpretation of the results. However, once FM is associated with low-grade inflammation, those dietary recommendations per se may not be anti-inflammatory enough.

\section{Trials status}

This is the first trial Protocol version, submitted on July 13, 2020. The trial is currently ongoing. Recruitment started on April 9, 2019, and will end in February 2021. We expect the end of the study to take place by April 2021.

\section{Abbreviations}

AGE: Advanced glycated product; BPI: Brief pain inventory;

Gl: Gastrointestinal; ESR: Eritrocyte sedimentation rate; EVA-Pain: Visual analog pain scale; EVA-GI: Visual analog scale from a list of common gastrointestinal and extraintestinal symptoms in FM; FIQ-R: Revised Fibromyalgia Impact Questionnaire; FM: Fibromyalgia; FODMAPs: Fermentable oligo-, di-, and monosaccharides and polyols; FSS: Fatigue severity survey; hs-CRP: Serum Creactive protein; IBS: Irritable bowel syndrome; IL: Interleukin; IPR: Instituto Português de Reumatologia; NSAID: Non-steroid anti-inflammatory drugs; PRO: Patient's reported outcomes; PSQI: Pittsburg sleep quality index; SF36: Short Form 36; SIBO: Small intestinal bacterial overgrowth; WHO: World Health Organization

\section{Supplementary Information}

The online version contains supplementary material available at https://doi. org/10.1186/s13063-021-05146-3.

Additional file 1. SPIRIT 2013 Checklist: Recommended items to address in a clinical trial protocol and related documents*

Additional file 2. Informed Consent Statement.

Additional file 3. FODMAPs diet support table for patients.

Additional file 4. Interleukin-8 collection procedures.

\section{Acknowledgements}

Not applicable.

\section{Organizational structure and responsibilities}

Trial Management Committee (TMC) members are ARS, JVP, MLS, and PP. Clinical Trial is monitored by the supervisors of the research work, namely JVP, MLS, and PP, for which periodic online meetings were held. Monitoring will be carried out during the implementation of the clinical trial, every 3 weeks.

Ethics Committee only evaluates the project at the initial moment. Data monitoring committee (DMC) is not needed, given that the overall risk for patients is low, and that one of the authors is not blinded. Given the nature of the study, problems that are detrimental to the participant are not anticipated, and therefore there is no interim analysis.

\section{Authors' contributions}

ARS, AB, MFM, JVP, PM, MLS, and PP conceived and planned the study design. ARS is the sponsor investigator and is carrying out the intervention. The sponsor is responsible for the study design, execution of the protocol, collection, management, analysis, and interpretation of the data, writing the reports, and submission to publication. Within the implementation of the study, JVP is the doctor responsible for enrolling participants. The sponsor is assigning participants to group interventions, applying the dietary intervention, monitoring participants' adherence to diet, and collecting the data. JVP, MLS, and PP are currently helping to supervise the project. ARS wrote the first draft of the manuscript and all authors revised it critically for important intellectual content. All authors provided critical feedback and helped shape the research, analysis, and manuscript. The authors read and approved the final manuscript.

\section{Funding}

This research did not receive any specific grant from funding agencies in the public, commercial, or not-for-profit sectors.

\section{Availability of data and materials}

Anonymized dataset will be stored in Google Drive, available to all authors. Any data required to support the protocol can be supplied on request.

\section{Declarations}

\section{Ethics approval and consent to participate}

This study was approved by the Ethics Committee of both IPR and Cooperativa de Ensino Superior Egas Moniz, with reference number 4/2020, and is being carried out in accordance with the Declaration of Helsinki (Declaration of 1975, revised in 2000). An informed consent is being given to all participants, after oral and written information about the study. Each participant is given a code and the anonymity and confidentiality of the data collected is ensured.

There were no amendments to the trial protocol. However, any modifications to the protocol will be documented in a breach report form, the ethical committee notified, and subsequently, clinical trial registry updated.

Results will be submitted for publication in a peer-reviewed journal. The datasets of the current study are available from the corresponding author on reasonable request.

\section{Consent for publication}

Not applicable.

\section{Competing interests}

The authors report no financial or personal conflicts of interest.

\section{Author details}

${ }^{1}$ Faculdade de Ciências da Nutrição e Alimentação, Universidade do Porto, Rua Dr. Roberto Frias, 4200-465 Porto, Portugal. ${ }^{2}$ Centro de Investigação Interdisciplinar Egas Moniz (CiiEM), Instituto Universitário Egas Moniz, Almada, Portugal. ${ }^{3}$ Instituto Português de Reumatologia (IPR), Rua Beneficiência 7, 1050-042 Lisbon, Portugal. " EPIUnit, Instituto de Saúde Pública, Universidade do Porto, Rua das Taipas, n 135, 4050-600 Porto, Portugal. ${ }^{5}$ Centro de Investigação em Atividade Física, Saúde e Lazer, Universidade do Porto, R. Dr. Plácido da Costa 91, 4200-450 Porto, Portugal. 
Received: 13 July 2020 Accepted: 22 February 2021

Published online: 09 March 2021

\section{References}

1. Clauw DJ. Fibromyalgia: an overview. Am J Med. 2009;122(12 Suppl):S3-S13.

2. Branco JC, et al. Prevalence of fibromyalgia: a survey in five European countries. Semin Arthritis Rheum. 2010;39(6):448-53.

3. Branco JC, et al. Prevalence of rheumatic and musculoskeletal diseases and their impact on health-related quality of life, physical function and mental health in Portugal: results from EpiReumaPt- a national health survey. RMD Open. 2016;2(1):e000166.

4. Wallace DJ, Hallegua DS. Fibromyalgia: the gastrointestinal link. Curr Pain Headache Rep. 2004:8(5):364-8.

5. Atzeni $F$, et al. Pain in systemic inflammatory rheumatic diseases. Best Pract Res Clin Rheumatol. 2015:29(1):42-52.

6. Triadafilopoulos G, Simms RW, Goldenberg DL. Bowel dysfunction in fibromyalgia syndrome. Dig Dis Sci. 1991;36(1):59-64.

7. Carding $\mathrm{S}$, et al. Dysbiosis of the gut microbiota in disease. Microb Ecol Health Dis. 2015;26:26191.

8. Marsh A, Eslick EM, Eslick GD. Does a diet low in FODMAPs reduce symptoms associated with functional gastrointestinal disorders? A comprehensive systematic review and meta-analysis. Eur J Nutr. 2016:55(3): 897-906.

9. Pimentel $\mathrm{M}$, et al. A link between irritable bowel syndrome and fibromyalgia may be related to findings on lactulose breath testing. Ann Rheum Dis. 2004;63(4):450-2

10. Goebel A, et al. Altered intestinal permeability in patients with primary fibromyalgia and in patients with complex regional pain syndrome. Rheumatology (Oxford). 2008;47(8):1223-7.

11. Marum AP, et al. A low fermentable oligo-di-mono saccharides and polyols (FODMAP) diet reduced pain and improved daily life in fibromyalgia patients. Scand J Pain. 2016;13:166-72.

12. Clauw DJ. Fibromyalgia and related conditions. Mayo Clin Proc. 2015;90(5): 680-92

13. Feng $B$, et al. Irritable bowel syndrome: methods, mechanisms, and pathophysiology. Neural and neuro-immune mechanisms of visceral hypersensitivity in irritable bowel syndrome. Am J Physiol Gastrointest Liver Physiol. 2012;302(10):G1085-98

14. Alam MS, et al. Interaction of Plasmodium vivax tryptophan-rich antigen PvTRAg38 with band 3 on human erythrocyte surface facilitates parasite growth. J Biol Chem. 2015;290(33):20257-72

15. Bazzichi L, et al. Cytokine patterns in fibromyalgia and their correlation with clinical manifestations. Clin Exp Rheumatol. 2007;25(2):225-30.

16. Uceyler N, Hauser W, Sommer C. Systematic review with meta-analysis: cytokines in fibromyalgia syndrome. BMC Musculoskelet Disord. 2011;12:245

17. Sanada K, et al. Effects of non-pharmacological interventions on inflammatory biomarker expression in patients with fibromyalgia: a systematic review. Arthritis Res Ther. 2015;17:272.

18. Cavicchia PP, et al. A new dietary inflammatory index predicts interval changes in serum high-sensitivity C-reactive protein. J Nutr. 2009;139(12): 2365-72.

19. Shivappa N, et al. Designing and developing a literature-derived, population-based dietary inflammatory index. Public Health Nutr. 2014;17(8): 1689-96.

20. Taneja V. Arthritis susceptibility and the gut microbiome. FEBS Lett. 2014; 588(22):4244-9.

21. Melnik BC. Milk--the promoter of chronic Western diseases. Med Hypotheses. 2009;72(6):631-9.

22. Straub $\mathrm{RH}$. Insulin resistance, selfish brain, and selfish immune system: an evolutionarily positively selected program used in chronic inflammatory diseases. Arthritis Res Ther. 2014;16(Suppl 2):S4.

23. Wahli W, Michalik L. PPARs at the crossroads of lipid signaling and inflammation. Trends Endocrinol Metab. 2012;23(7):351-63.

24. Suen $\mathrm{J}$, et al. Effect of flavonoids on oxidative stress and inflammation in adults at risk of cardiovascular disease: a systematic review. Healthcare. 2016;4(3):69.

25. Donaldson MS, Speight N, Loomis S. Fibromyalgia syndrome improved using a mostly raw vegetarian diet: an observational study. BMC Complement Altern Med. 2001;1:7.

26. Kaartinen $\mathrm{K}$, et al. Vegan diet alleviates fibromyalgia symptoms. Scand J Rheumatol. 2000;29(5):308-13.
27. Shapiro JR, Anderson DA, Danoff-Burg S. A pilot study of the effects of behavioral weight loss treatment on fibromyalgia symptoms. J Psychosom Res. 2005;59(5):275-82.

28. Senna MK, et al. Effect of weight reduction on the quality of life in obese patients with fibromyalgia syndrome: a randomized controlled trial. Clin Rheumatol. 2012;31(11):1591-7.

29. Silva $A R$, et al. Dietary interventions in fibromyalgia: a systematic review. Ann Med. 2019:51:1-29.

30. Organization, W.H. Healthy diet, vol. 394; 2018. p. 1-6.

31. Calder PC, et al. Inflammatory disease processes and interactions with nutrition. Br J Nutr. 2009;101(Suppl 1):S1-45.

32. Kontogianni MD, Zampelas A, Tsigos C. Nutrition and inflammatory load. Ann N Y Acad Sci. 2006:1083:214-38.

33. Ruiz-Nunez B, et al. Lifestyle and nutritional imbalances associated with Western diseases: causes and consequences of chronic systemic low-grade inflammation in an evolutionary context. J Nutr Biochem. 2013;24(7):1183-201.

34. Zhong $D$, et al. The role of gut microbiota in the pathogenesis of rheumatic diseases. Clin Rheumatol. 2018;37(1):25-34.

35. Belkaid $Y$, Hand TW. Role of the microbiota in immunity and inflammation. Cell. 2014;157(1):121-41.

36. Minihane AM, et al. Low-grade inflammation, diet composition and health: current research evidence and its translation. Br J Nutr. 2015;114(7):999-1012.

37. Hollon J, et al. Effect of gliadin on permeability of intestinal biopsy explants from celiac disease patients and patients with non-celiac gluten sensitivity. Nutrients. 2015;7(3):1565-76.

38. Fasano A. Zonulin, regulation of tight junctions, and autoimmune diseases. Ann N Y Acad Sci. 2012;1258:25-33.

39. O'Toole A, Korzenik J. Environmental triggers for IBD. Curr Gastroenterol Rep. 2014;16(7):396.

40. Kunz C, Lonnerdal B. Human milk proteins: separation of whey proteins and their analysis by polyacrylamide gel electrophoresis, fast protein liquid chromatography (FPLC) gel filtration, and anion-exchange chromatography. Am J Clin Nutr. 1989;49(3):464-70.

41. Pal S, et al. Milk intolerance, beta-casein and lactose. Nutrients. 2015;7(9): 7285-97.

42. Brooke-Taylor $\mathrm{S}$, et al. Systematic review of the gastrointestinal effects of $A 1$ compared with A2 beta-casein. Adv Nutr. 2017:8(5):739-48.

43. UI Haq MR, et al. Comparative evaluation of cow beta-casein variants (A1/ A2) consumption on Th2-mediated inflammatory response in mouse gut. Eur J Nutr. 2014:53(4):1039-49.

44. Kim JA, Wei Y, Sowers JR. Role of mitochondrial dysfunction in insulin resistance. Circ Res. 2008;102(4):401-14.

45. Della Corte KW, et al. Effect of dietary sugar intake on biomarkers of subclinical inflammation: a systematic review and meta-analysis of intervention studies. Nutrients. 2018;10(5):606

46. Haroon $\mathrm{E}$, Miller AH. Inflammation effects on brain glutamate in depression: mechanistic considerations and treatment implications. Curr Top Behav Neurosci. 2017;31:173-98.

47. Laudisi $F$, et al. The food additive maltodextrin promotes endoplasmic reticulum stress-driven mucus depletion and exacerbates intestinal inflammation. Cell Mol Gastroenterol Hepatol. 2019:7(2):457-73.

48. Guilbaud A, et al. How can diet affect the accumulation of advanced glycation end-products in the human body? Foods. 2016:5(4):84.

49. Uribarri J, et al. Advanced glycation end products in foods and a practical guide to their reduction in the diet. J Am Diet Assoc. 2010; 110(6):911-6 e12.

50. Teodorowicz $M$, van Neerven J, Savelkoul H. Food processing: the influence of the maillard reaction on immunogenicity and allergenicity of food proteins. Nutrients. 2017;9(8):835

51. Luevano-Contreras C, Chapman-Novakofski K. Dietary advanced glycation end products and aging. Nutrients. 2010;2(12):1247-65.

52. Tabrizi $R$, et al. The effects of resveratrol supplementation on biomarkers of inflammation and oxidative stress among patients with metabolic syndrome and related disorders: a systematic review and meta-analysis of randomized controlled trials. Food Funct. 2018;9(12):6116-28

53. Carlsen $\mathrm{MH}$, et al. The total antioxidant content of more than 3100 foods, beverages, spices, herbs and supplements used worldwide. Nutr J. 2010;9:3.

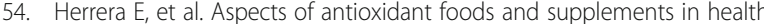
and disease. Nutr Rev. 2009;67(Suppl 1):S140-4.

55. Organization, W.H. Healthy diet. 2018; Available from: https://www.who.int/ en/news-room/fact-sheets/detail/healthy-diet. 
56. Costa C, et al. Psychometric properties of the Revised Fibromyalgia Impact Questionnaire (FIQR) - a contribution to the Portuguese validation of the scale. Acta Reumatol Port. 2016;41(3):240-50.

57. Price $\mathrm{DD}$, et al. The validation of visual analogue scales as ratio scale measures for chronic and experimental pain. Pain. 1983;17(1):45-56.

58. Boonstra AM, et al. Reliability and validity of the visual analogue scale for disability in patients with chronic musculoskeletal pain. Int J Rehabil Res. 2008;31(2):165-9.

59. Valente MAF, Ribeiro JLP, Jensen MP. Further validation of a portuguese version of the brief pain inventory interference scale. Clín Salud. 2012;23(1): 89-96.

60. Keller $\mathrm{S}$, et al. Validity of the brief pain inventory for use in documenting the outcomes of patients with noncancer pain. Clin J Pain. 2004;20(5):309-18.

61. Bengtsson M, Ohlsson B, Ulander K. Development and psychometric testing of the visual analogue scale for irritable bowel syndrome (VAS-IBS). BMC Gastroenterol. 2007;7:16.

62. Bengtsson $\mathrm{M}$, et al. Further validation of the visual analogue scale for irritable bowel syndrome after use in clinical practice. Gastroenterol Nurs. 2013:36(3):188-98.

63. Ferreira, P.L., [Development of the Portuguese version of MOS SF-36. Part II --validation tests]. Acta Medica Port. 2000;13(3):119-27.

64. Fredheim $\mathrm{OM}$, et al. Validation and comparison of the health-related quality-of-life instruments EORTC QLQ-C30 and SF-36 in assessment of patients with chronic nonmalignant pain. J Pain Symptom Manag. 2007; 34(6):657-65.

65. Laranjeira CA. Translation and adaptation of the fatique severity scale for use in Portugal. Appl Nurs Res. 2012;25(3):212-7.

66. Del Rio Joao KA, et al. Validation of the Portuquese version of the Pittsburgh Sleep Quality Index (PSQI-PT). Psychiatry Res. 2017;247:225-9.

67. Slim M, et al. The effects of a gluten-free diet versus a hypocaloric diet among patients with fibromyalgia experiencing gluten sensitivity-like symptoms: a pilot, open-label randomized clinical trial. J Clin Gastroenterol. 2017;51(6):500-7.

68. Vellisca MY, Latorre Jl. Monosodium glutamate and aspartame in perceived pain in fibromyalgia. Rheumatol Int. 2014;34(7):1011-3.

\section{Publisher's Note}

Springer Nature remains neutral with regard to jurisdictional claims in published maps and institutional affiliations.

Ready to submit your research? Choose BMC and benefit from:

- fast, convenient online submission

- thorough peer review by experienced researchers in your field

- rapid publication on acceptance

- support for research data, including large and complex data types

- gold Open Access which fosters wider collaboration and increased citations

- maximum visibility for your research: over $100 \mathrm{M}$ website views per year

At $\mathrm{BMC}$, research is always in progress.

Learn more biomedcentral.com/submissions 